gametocytes rescued development of ookinetes to untreated control levels when grown in filtered Esp_Z culture medium (Fig. 3B), and parasite development in the presence of replicating Esp_Z was rescued by vitC in a dose-dependent fashion (Fig. 3C). Furthermore, reduced glutathione, another potent antioxidant, also rescued in vitro ookinete formation in the presence of $E s p \_Z$ (fig. S7). We showed that vertebrate leukocytes were not responsible for the observed in vitro ookinete inhibition (fig. S8). More important, supplementing an infectious blood meal with vitC did not affect parasite numbers in the absence of $E s p \_Z$ but rescued $P$. falciparum ookinete development twofold in the lumen of $A$. gambiae midguts when they were cofed with Esp_Z (Fig. 3D). The significant, yet incomplete, rescue of ookinete development with higher bacterial concentrations could be attributed to a variety of factors such as insufficient concentrations of antioxidant to neutralize the higher amount of bacterially produced ROS, excretion of substantial amounts of antioxidant by mosquito diuresis, an intimate association between bacteria and parasites that may not enable detoxification of ROS before parasite inhibition, or the loss of antioxidant activity from prolonged exposure in the digestive environment of the midgut. Antioxidant concentrations higher than $10 \mathrm{mM}$ in the blood meal interfered with mosquito feeding propensity.

Genotypic analyses of laboratory and wild mosquito populations have suggested that a dominant refractory phenotype is associated with innate immunity and that Plasmodium infection is a result of immune failure (21-23). Our studies show a mechanism of Plasmodium inhibition that does not involve the mosquito-derived innate immune response, and they support the idea that the native microflora of Anopheles mosquitoes plays a cru- cial role in refractoriness to Plasmodium infection and will therefore influence transmission success to humans.

Bacteria of the genus Enterobacter have been isolated from many anopheline mosquito species in diverse geographic regions $(3,5,24)$. We show that mosquitoes do not become infected with Plasmodium parasites when exposed to an Enterobacter bacterium isolated from wild mosquito populations in southern Zambia, and we show that inhibition of parasite development can be mediated by bacterial generation of ROS. Although Esp_Z was isolated from a single collection made during one rainy season, $25 \%$ of mosquitoes collected harbored the strain. It may be possible to manipulate the composition of the midgut microbial flora in wild mosquitoes to increase the prevalence of Esp_Z or other naturally inhibitory bacteria as part of an integrated malaria control strategy.

References and Notes

1. Y. Alavi et al., Int. J. Parasitol. 33, 933 (2003).

2. J. A. Vaughan, B. H. Noden, J. C. Beier, Am. J. Trop. Med. Hyg. 51, 233 (1994).

3. L. Gonzalez-Ceron, F. Santillan, M. H. Rodriguez, D. Mendez, J. E. Hernandez-Avila, J. Med. Entomol. 40, 371 (2003)

4. C. B. Pumpuni, M. S. Beier, J. P. Nataro, L. D. Guers, J. R. Davis, Exp. Parasitol. 77, 195 (1993).

5. C. B. Pumpuni, J. Demaio, M. Kent, J. R. Davis, J. C. Beier, Am. J. Trop. Med. Hyg. 54, 214 (1996).

6. Y. Dong, F. Manfredini, G. Dimopoulos, PLoS Pathog. 5, e1000423 (2009)

7. S. Meister et al., PLoS Pathog. 5, e1000542 (2009).

8. L. S. Garver, Y. Dong, G. Dimopoulos, PLoS Pathog. 5, e1000335 (2009).

9. Y. Dong et al., PLoS Pathog. 2, e52 (2006).

10. S. Kumar, A. Molina-Cruz, L. Gupta, J. Rodrigues, C. Barillas-Mury, Science 327, 1644 (2010).

11. Materials and methods are available as supporting material on Science online.
12. C. M. Cirimotich, Y. Dong, L. S. Garver, S. Sim, G. Dimopoulos, Dev. Comp. Immunol. 34, 387 (2010).

13. J. A. Vaughan, B. H. Noden, ]. C. Beier, J. Parasitol. 78, 716 (1992)

14. S. Meister et al., Proc. Natl. Acad. Sci. U.S.A. 102, 11420 (2005)

15. K. van Dijk, E. B. Nelson, Appl. Environ. Microbiol. 66, 5340 (2000).

16. S. Kumar et al., Proc. Natl. Acad. Sci. U.S.A. 100, 14139 (2003).

17. S. Luckhart, Y. Vodovotz, L. Cui, R. Rosenberg, Proc. Natl. Acad. Sci. U.S.A. 95, 5700 (1998).

18. A. Molina-Cruz et al., J. Biol. Chem. 283, 3217 (2008)

19. T. M. L. Peterson, A. J. Gow, S. Luckhart, Free Radic. Biol. Med. 42, 132 (2007).

20. A. Mai-Prochnow et al., J. Bacteriol. 190, 5493 (2008)

21. S. A. Blandin et al., Science 326, 147 (2009).

22. 0. Niaré et al., Science 298, 213 (2002).

23. M. M. Riehle et al., Science 312, 577 (2006).

24. 0. Terenius et al., J. Med. Entomol. 45, 172 (2008).

Acknowledgments. This work has been supported by the National Institutes of Health/National Institute of Allergy and Infectious Disease R01Al061576 and a Johns Hopkins Malaria Research Institute (JHMRI) Pilot Grant (to G.D.), the Bloomberg Family Foundation, the Calvin A. and Helen H. Lang fellowship (to C.M.C.), a JHMRI postdoctoral fellowship (to J.S.-N.), and a fellowship from the NSF (to A.M.C.). The authors thank the mosquito collection team at the Malaria Institute at Macha, Zambia; the JHMRI Parasitology and Insectary Core facilities; Sanaria Inc.; E. Nelson (Cornell University) for providing mutant bacteria strains; and D. McClellan for editorial services. We performed all experiments according to Johns Hopkins Institutional Animal Care and Use Committee guidelines. Human landing catches were performed according to the approved protocol UNZA REC 011-02-04 with consent of participants. GenBank accession numbers generated for bacterial 165 rDNA sequences are listed in table S1, SOM.

\section{Supporting Online Material}

www.sciencemag.org/cgi/content/full/332/6031/855/DC1

Materials and Methods

Figs. S1 to S8

Tables S1 to S3

References and Notes

13 December 2010; accepted 6 April 2011 10.1126/science. 1201618

\title{
Preserved Feedforward But Impaired Top-Down Processes in the Vegetative State
}

\author{
Melanie Boly, ${ }^{1,2 *}$ Marta Isabel Garrido, ${ }^{2}$ Olivia Gosseries, ${ }^{1}$ Marie-Aurélie Bruno, ${ }^{1}$ \\ Pierre Boveroux, ${ }^{3}$ Caroline Schnakers, ${ }^{1}$ Marcello Massimini, ${ }^{4}$ Vladimir Litvak, ${ }^{2}$ \\ Steven Laureys, ${ }^{1}$ Karl Friston $^{2}$
}

Frontoparietal cortex is involved in the explicit processing (awareness) of stimuli. Frontoparietal activation has also been found in studies of subliminal stimulus processing. We hypothesized that an impairment of top-down processes, involved in recurrent neuronal message-passing and the generation of long-latency electrophysiological responses, might provide a more reliable correlate of consciousness in severely brain-damaged patients, than frontoparietal responses. We measured effective connectivity during a mismatch negativity paradigm and found that the only significant difference between patients in a vegetative state and controls was an impairment of backward connectivity from frontal to temporal cortices. This result emphasizes the importance of top-down projections in recurrent processing that involve high-order associative cortices for conscious perception.

$\mathrm{T}$ he vegetative state (VS) is defined by preserved arousal, in the absence of any behavioral signs of awareness (1). In contrast, patients in a minimally conscious state (MCS) show nonreflexive and purposeful behaviors but are unable to communicate (2). Because the clin- ical diagnosis of these patients is extremely difficult (3), neuroimaging experiments have tried to establish accurate biomarkers of consciousness level in VS and MCS. These patients also provide a lesion-deficit model in the quest for neural correlates of consciousness in the human brain (4). The conscious perception of external stimuli requires activation of frontoparietal cortices, in addition to activity in low-level specialized cortices (5-7). However, frontoparietal activation can also be found during subliminal stimulus processing $(8,9)$. Current evidence points to long-latency evoked event-related potential (ERP) components, involving frontoparietal cortices, as a reliable neu-

${ }^{1}$ Coma Science Group, Cyclotron Research Centre and Neurology Department, University of Liège and CHU Sart Tilman Hospital, 4000 Liège, Belgium. ${ }^{2}$ Wellcome Trust Centre for Neuroimaging, Institute of Neurology, University College London, London WC1N 1P], UK. ${ }^{3}$ Anesthesiology Department, University of Liège and CHU Sart Tilman Hospital, 4000 Liège, Belgium. ${ }^{4}$ Department of Clinical Sciences, "Luigi Sacco," University of Milan, 20157 Milan, Italy.

*To whom correspondence should be addressed. E-mail: mboly@ulg.ac.be 
A

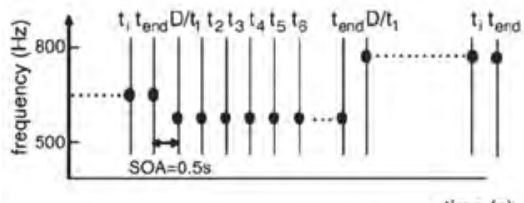

$\mathrm{D} / \mathrm{t}_{1}=$ deviant

$t_{i}=$ trial $i, 1 \leq i \leq 11$
B

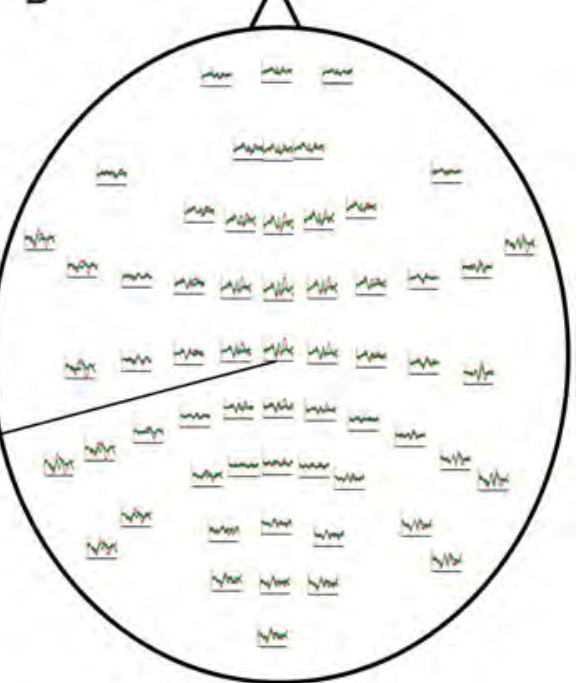

Fig. 1. Design and ERP responses elicited in a roving paradigm. (A) The experimental design used a sporadically changing standard stimulus (auditory tone). The first presentation of a novel tone is a deviant $\mathrm{D}=t_{1}$ that becomes a standard, through repetition; $t_{2}, \ldots, t_{\text {end }}$. In this paradigm, deviants and standard have exactly the same physical properties. (B) Grand-mean (averaged over control subjects) ERP responses to the first and 11th tone presentations: the established "standard" ( $t_{11}$ in green) and deviant tone ( $t_{1}$ in red) overlaid on a scalp map of 60 electroencephalogram electrodes. (C) ERP responses to standard and deviant tones at channel $\mathrm{Cz}$ (central). Deviant corresponds to responses elicited by the first tone presentation or to oddball events. Standard corresponds to the 11th repetition of the same tone. [Adapted from a figure in M. I. Garrido et al. (22), with permission from Elsevier] ronal marker for conscious perception in healthy controls (10). Although feed-forward connectivity is sufficient to generate short-latency ERP components, long-latency components are mediated by backward connections (11). This suggests that the level of consciousness may rest on the integrity of backward (top-down) connectivity. We used a mismatch negativity (MMN) paradigm, which elicits well-characterized (preattentive) responses (12). The MMN correlates inversely with consciousness level during anesthesia (13) and with depth of sleep (14); although some studies report negative findings (15), MMN deficits have also been associated with the level of consciousness in MCS and VS (16) [see supporting online material (SOM) text]. We predicted that both longlatency components and backward connectivity would correlate with level of consciousness. We tested this hypothesis quantitatively, using Dynamic Causal Modeling (DCM), which allows for inferences about the neuronal architectures that generate hemodynamic or electromagnetic signals $(17,18)$. DCM uses a generative model of how electrophysiological signals are produced at the neuronal level; it employs neural mass models and Bayesian statistics to infer the neuronal mechanisms (effective connectivity) underlying observed evoked electrophysiological responses $(19,20)$. This method has been validated in a
Fig. 2. Spatiotemporal grand-mean difference between standard and deviants correlates with the level of consciousness. The two-dimensional scalp topographies are interpolated from the 60 channels. (A) Statistical parametric maps of differential group responses in controls, MCS patients, and VS patients. For display purposes, instantaneous T maps are displayed without threshold and with threshold at $P<0.001$ (uncorrected) in the three populations. Color scales correspond respectively to $\mathrm{T}$ values or to significant (in black) voxels. (B) Statistical parametric maps showing scalp regions where there is a significant interaction between response amplitude and consciousness level (with the threshold at $P<$ 0.05; family-wise error corrected) over different time windows. Significant effects were found over temporal and frontal sensors in the range of 115 to $395 \mathrm{~ms}$.

A Controls

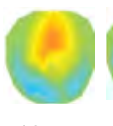
. $(18)$ 4 Qv (2) $16 \mathrm{~ms}$ $48 \mathrm{~ms} 72 \mathrm{~ms}$ $116 \mathrm{~ms}$
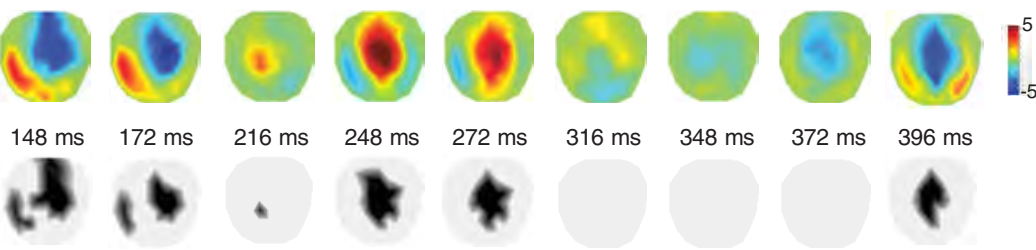

$372 \mathrm{~ms}$
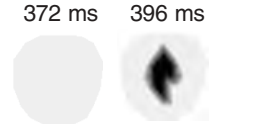

MCS
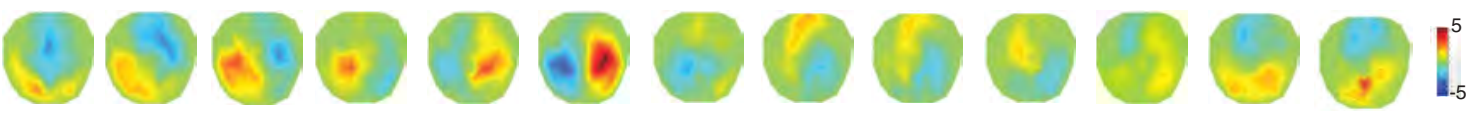

$16 \mathrm{~ms}$
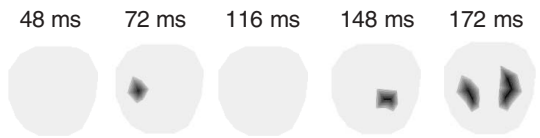

$216 \mathrm{~ms}$
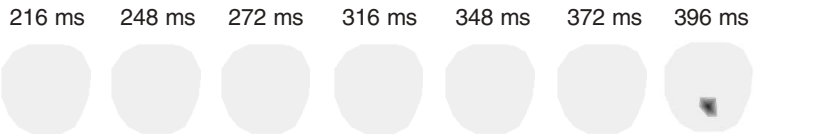

\section{vS}
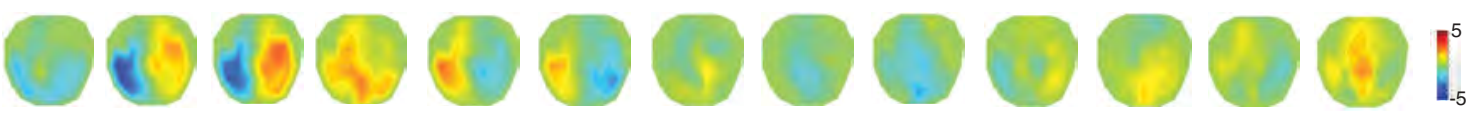

$216 \mathrm{~ms}$

$248 \mathrm{~ms}$

$272 \mathrm{~ms}$

$316 \mathrm{~ms}$

$348 \mathrm{~ms}$

$372 \mathrm{~ms}$

$396 \mathrm{~ms}$

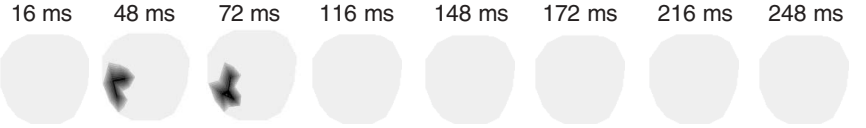

B

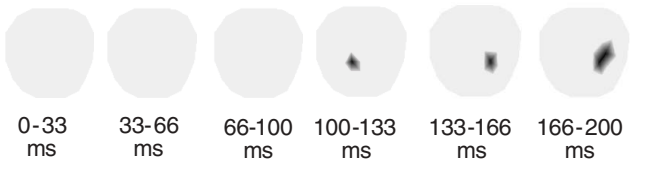

Level of consciousness effect

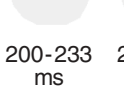

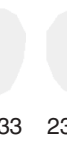

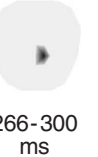



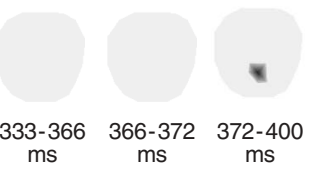


number of electrophysiological studies $(11,21-23)$ and provides an efficient way to map from observed evoked potential patterns to causative neuronal mechanisms (24).

The present study used DCM of event-related potentials to quantify effective connectivity in backward and forward connections at two hierarchical cortical levels (i.e., in temporal and frontal cortices), during auditory processing in healthy subjects and MCS and VS patients. We used a roving oddball paradigm (Fig. 1), as in previous DCM studies $(22,23)$ (SOM text). The functional anatomy engaged by this paradigm involves both forward and backward connections in a fronto-temporal cortical network (22). Highdensity ERP recordings were acquired in 21 braindamaged patients (8 VS and $13 \mathrm{MCS}$ ) and 22 healthy controls (25). ERP data analysis, performed with statistical parametric mapping, tested the differential processing of standard and deviant sounds (25). Scalp-level group analyses detected longlatency components with a central topography in both controls and patients in a MCS $(P<0.05$, family-wise error corrected). Peak latencies for the MMN were $170 \mathrm{~ms}$ in controls and $175 \mathrm{~ms}$ in patients in a MCS. Although the MMN was the most significant in MCS, the P3 component

A DCM models

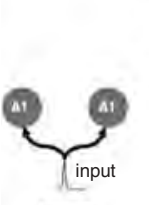

1
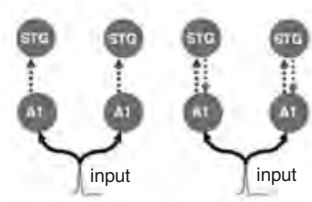

2

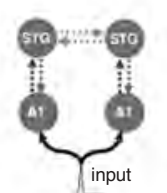

3
4
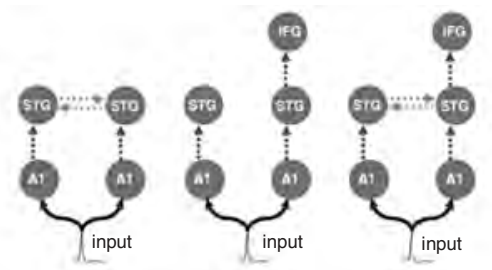

5

7

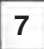

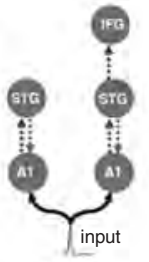

8
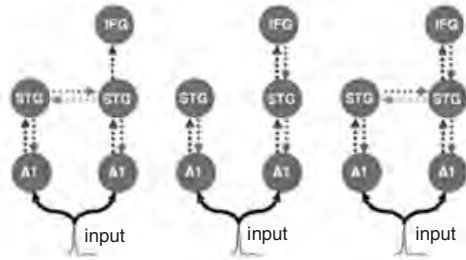

vs

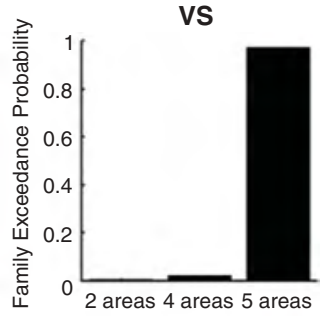

\section{Family inference - type of connections}
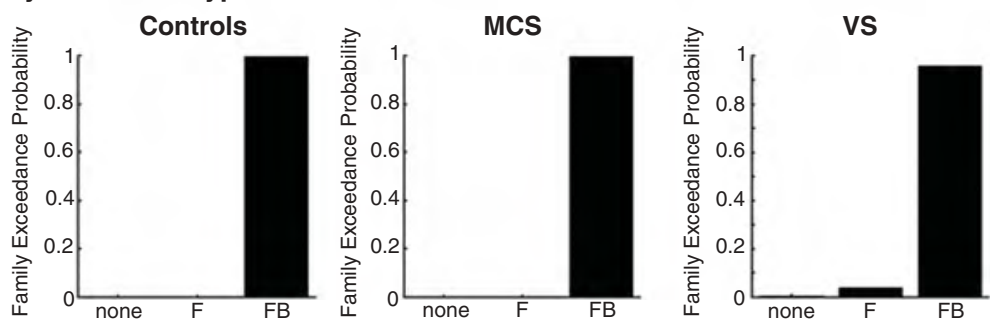

\section{E Source activity estimates}

Control

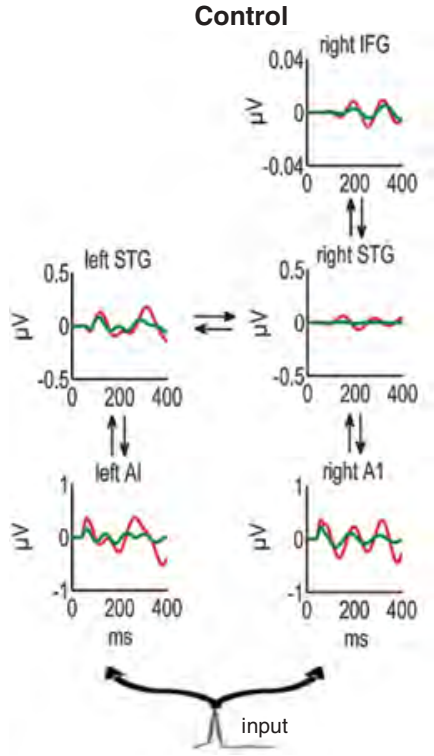

MCS

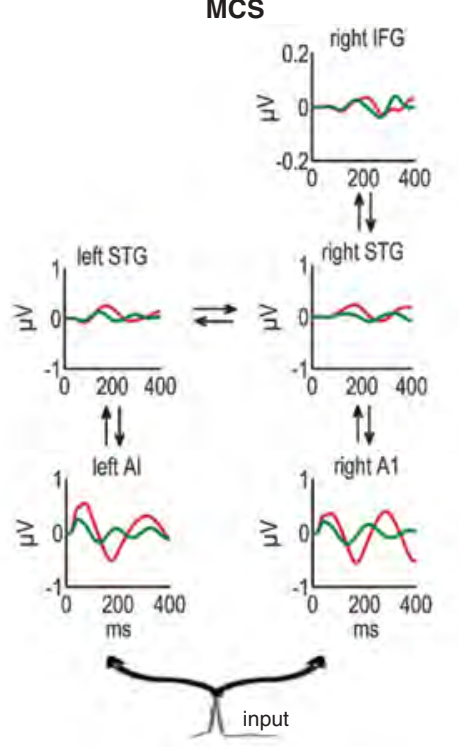

VS

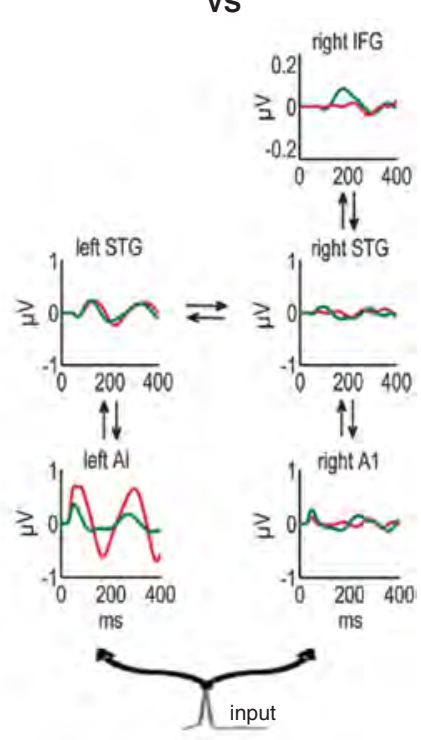

D

Population-level best model

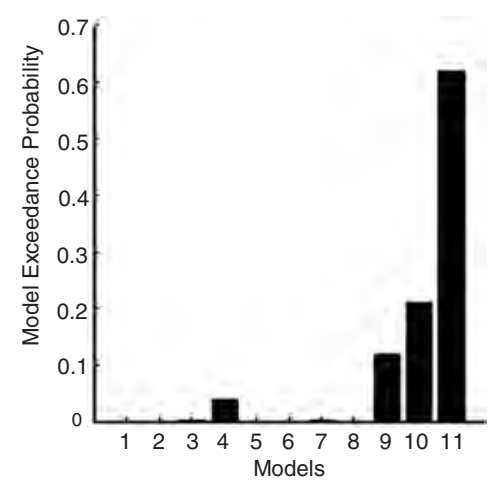

Fig. 3. Model specification. (A) The 11 DCMs used for Bayesian model comparison. Each model receives (parameterized) subcortical input at the $A 1$ sources, which elicit transient perturbations in the remaining sources. (B to $\mathbf{C}$ ) Familywise Bayesian model selection was used to establish the neuronal network architecture in each population. In the three populations studied, the best models included a frontal region (B) and the presence of both backward and forward connections (C). (D) Random effects Bayesian model selection showed that the fully connected model (model 11) had the greatest evidence and was selected for subsequent quantitative analysis of effective connectivity across the three populations. (E) Source activity estimates for standard (green) and deviant (red) tones according to model 11 in an exemplar control and MCS and VS subjects. 
(peak latency at $250 \mathrm{~ms}$ ) was the most significant in controls. Only posterior early components (peak at $55 \mathrm{~ms}$ ) could be seen in VS (Fig. 2). An analysis of covariance (ANCOVA) confirmed a significant interaction between level of consciousness and stimulus type on long-latency responses (peak latencies at 115, 175, 265, and $395 \mathrm{~ms}, P<0.05$, family-wise error corrected) across the three groups (Fig. 2).

Although the architecture of the network involved in the generation of MMN responses has been studied extensively in healthy volunteers (26) (SOM text), the involvement of frontal cortex, via its forward and backward connections, has yet to be established in brain-damaged patients. We thus performed a group-level Bayesian model comparison to identify the network architecture that best explained the responses of patients. Bayesian model selection (BMS) compared 11 models, which differed in the areas and connections involved (Fig. 3). Random effects familylevel analysis showed that models including a frontal source with both forward and backward connections could best explain ERP responses in MCS and VS. For quantitative connectivity analysis, a second (random-effects) BMS procedure was applied to the 11 models, across all three groups (25). The fully connected model (model 11) had again the greatest evidence (Fig. 3). The corresponding source activity estimates in representative controls and MCS and VS subjects are also shown in Fig. 3. To assess differences between controls and patients, we compared the connectivity estimates (from the best model) using simple $t$ tests (25). The only significant effect of the level of consciousness (differences between healthy volunteers, MCS subjects, and VS patient populations) was on the backward connection from frontal to superior temporal cortex (corrected $P=0.012$ ), with no detectible differences in forward and backward processing in temporal cortex (Fig. 4). This backward connection was significantly impaired in VS patients as compared with controls $(P=0.002)$. MCS patients showed preserved top-down connectivity as compared with VS patients $(P=0.001)$ and were not significantly different from controls $(P>0.05)$ (Fig. 4).

Our main result is that, although the frontal cortex is still involved in the generation of the
ERP in a VS, the main abnormality is in recurrent processing between higher-order cortices, owing to impaired backward connections from frontal to temporal cortices. Our analyses suggest that the (possibly diverse) pathophysiological causes of VS find a common expression in reducing topdown influences from frontal to temporal areas. Further research is required to establish the precise molecular or physiological mechanisms underlying these observations. In contrast to VS patients, MCS patients exhibited long-latency components in the scalp ERP and near-normal recurrent effective connectivity with higher-order cortices. This group difference cannot be attributable to differences in vigilance, as all patients were maintained in the same state of wakefulness throughout the experiment (25). Although forward connections are certainly needed for normal stimulus processing, these results suggest that the integrity of backward connections, or top-down processes, may be necessary for conscious perception. These findings stress the importance of recurrent processing in higher-order associative areas in the generation of conscious perception and do not support the view that recurrent processing in sensory cortex can be equated with consciousness (27). In contrast, our results suggest that recursive processing in high-order cortical areas is necessary for the generation of conscious perception (5). This would be mandated by the maintenance of high levels of integrated information, thought to be important for consciousness (28). A hierarchical recurrent cortical organization is also required by predictive coding formulations of the Bayesian brain hypothesis, which regards perception as inference on the causes of external stimuli (29). Under this perspective, VS patients are unable to elaborate (top-down) predictions of their sensorium, resulting (phenomenologically) in an impoverished percept and (physiologically) the absence of long-latency (endogenous) ERP components. Finally, our biologically plausible DCM shows that a selective impairment of a single (backward) connection accounts for widespread differences in the distributed responses we found between VS patients and healthy volunteers. This fits with the pervasive effects of recurrent processing on ERP amplitudes observed in healthy volunteers (a phenomenon referred to as "igni-

Fig. 4. Quantitative effective connectivity analysis revealed that the only significant difference between VS patients and controls was an impairment of backward connectivity from frontal to temporal cortex. MCS subjects showed significantly preserved connectivity compared with VS subjects and were not significantly different from controls.
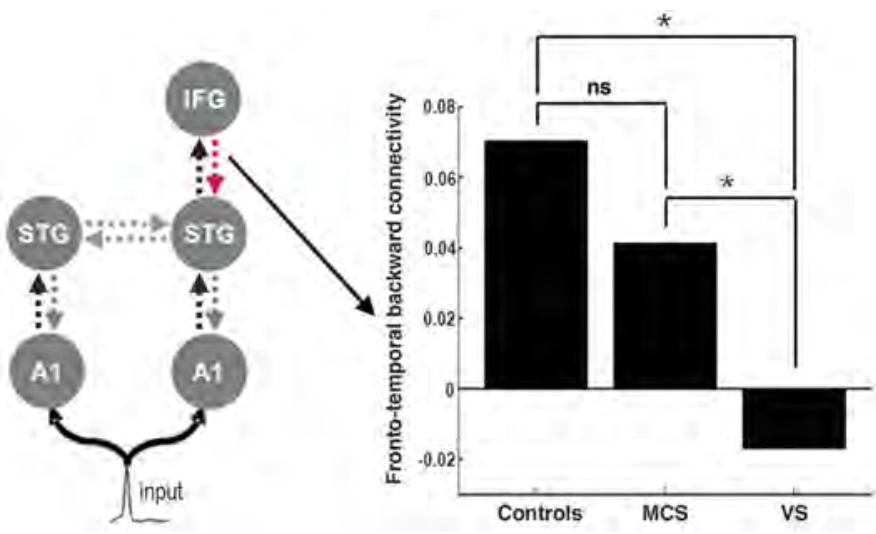

tion") (10). Long-lasting slow ERP responses, such as K-complexes, can be observed during slowwave sleep (30). Cortical connectivity analysis could be thus more specific than scalp-level analyses in terms of detecting the architectural correlates of conscious level. On the other hand, our analyses cannot say whether a failure of backward connectivity from the frontal region is a cause or consequence of processing that underlies consciousness (SOM text). What we can say is that a specific (top-down) failure of effective connectivity is implicated in consciousness and may play a necessary role.

In summary, our findings suggest that a selective disruption of top-down processes from high levels of a cortical hierarchy can lead to loss of consciousness in brain-damaged patients, and can clearly differentiate VS from MCS. In addition to its neuroscientific relevance, the present approach could constitute a new diagnostic tool to quantify the level of consciousness electrophysiologically at the patients' bedside. Further studies should also complement these findings in conditions such as sleep, epilepsy, or anesthesia-induced unconsciousness.

\section{References and Notes}

1. S. Laureys, A. M. Owen, N. D. Schiff, Lancet Neurol. 3, 537 (2004).

2. ]. T. Giacino et al., Neurology 58, 349 (2002).

3. C. Schnakers et al., BMC Neurol. 9, 35 (2009).

4. S. Laureys, Trends Cogn. Sci. 9, 556 (2005).

5. S. Dehaene, J. P. Changeux, L. Naccache, J. Sackur, C. Sergent, Trends Cogn. Sci. 10, 204 (2006).

6. G. Rees, G. Kreiman, C. Koch, Nat. Rev. Neurosci. 3, 261 (2002).

7. M. Boly et al., Arch. Neurol. 61, 233 (2004).

8. M. T. Diaz, G. McCarthy, J. Cogn. Neurosci. 19, 1768 (2007).

9. Q. Luo et al., Cereb. Cortex 19, 1896 (2009).

10. A. Del Cul, S. Baillet, S. Dehaene, PLoS Biol. 5, e260 (2007).

11. M. I. Garrido, ]. M. Kilner, S. J. Kiebel, K. J. Friston, Proc. Natl. Acad. Sci. U.S.A. 104, 20961 (2007)

12. R. Näätänen, P. Paavilainen, T. Rinne, K. Alho, Clin. Neurophysiol. 118, 2544 (2007).

13. W. Heinke et al., Anesthesiology 100, 617 (2004).

14. P. Ruby, A. Caclin, S. Boulet, C. Delpuech, D. Morlet, J. Cogn. Neurosci. 20, 296 (2008).

15. C. Fischer, ]. Luaute, D. Morlet, Clin. Neurophysiol. 121, 1032 (2010)

16. V. J. Wijnen, G. J. van Boxtel, H. J. Eilander, B. de Gelder, Clin. Neurophysiol. 118, 597 (2007).

17. K. J. Friston, L. Harrison, W. Penny, Neuroimage 19. 1273 (2003).

18. O. David et al., Neuroimage 30, 1255 (2006).

19. O. David, L. Harrison, K. J. Friston, Neuroimage 25, 756 (2005).

20. S. J. Kiebel, M. I. Garrido, R. Moran, C. C. Chen, K. J. Friston, Hum. Brain Mapp. 30, 1866 (2009).

21. M. I. Garrido, ]. M. Kilner, S. J. Kiebel, K. E. Stephan, K. J. Friston, Neuroimage 36, 571 (2007).

22. M. I. Garrido et al., Neuroimage 42, 936 (2008).

23. M. I. Garrido et al., Neuroimage 48, 269 (2009).

24. K. J. Friston, Science 326, 399 (2009).

25. Materials and methods are available as supporting material on Science Online.

26. M. I. Garrido, J. M. Kilner, K. E. Stephan, K. J. Friston Clin. Neurophysiol. 120, 453 (2009).

27. V. A. Lamme, Trends Cogn. Sci. 10, 494 (2006).

28. D. Balduzzi, G. Tononi, PLOS Comput. Biol. 4, e1000091 (2008).

29. K. Friston, Nat. Rev. Neurosci. 11, 127 (2010)

30. M. Roth, ]. Shaw, ]. Green, Electroencephalogr. Clin. Neurophysiol. 8, 385 (1956). 
Acknowledgments. This work was supported by the Belgian Fonds National de la Recherche Scientifique (FNRS) European Commission, Mind Science Foundation, McDonnell Foundation, French-Speaking Community Concerted Research Action (ARC 06/11-340), Fondation Léon Frédéricq, and National Institutes of Health.

M.-A.B. and O.G. are Research Fellows, M.B. and C.S.
Postdoctoral Fellows, and S.L. Senior Research Associate at the FNRS. M.I.G., V.L., and K.F. are supported by the Wellcome Trust.

Supporting Online Material

www.sciencemag.org/cgi/content/full/332/6031/858/DC1 Materials and Methods
SOM Text

Fig. S1

Table S1

References

22 December 2010; accepted 6 April 2011

10.1126/science. 1202043

\section{Improved Learning in a Large-Enrollment Physics Class}

\author{
Louis Deslauriers, ${ }^{1,2}$ Ellen Schelew, ${ }^{2}$ Carl Wieman* ${ }^{*} \ddagger$
}

We compared the amounts of learning achieved using two different instructional approaches under controlled conditions. We measured the learning of a specific set of topics and objectives when taught by 3 hours of traditional lecture given by an experienced highly rated instructor and 3 hours of instruction given by a trained but inexperienced instructor using instruction based on research in cognitive psychology and physics education. The comparison was made between two large sections $(N=267$ and $N=271)$ of an introductory undergraduate physics course. We found increased student attendance, higher engagement, and more than twice the learning in the section taught using research-based instruction.

$\mathrm{T}$ he traditional lecture approach remains the prevailing method for teaching science at the postsecondary level, although there are a growing number of studies indicating that other instructional approaches are more effective (1-8). A typical study in the domain of physics demonstrates how student learning is improved from one year to the next when an instructor changes his or her approach, as measured by standard concept-based tests such as the Force Concept Inventory (9) or the instructor's own exams. In our studies of two full sessions of an advanced quantum mechanics class taught either by traditional or by interactive learning style, students in the interactive section showed improved learning, but both sections, interactive and traditional, showed similar retention of learning 6 to 18 months later (10). Here, we compare learning produced by two contrasting instructional methods in a large-enrollment science course. The control group was lectured by a motivated faculty member with high student evaluations and many years of experience teaching this course. The experimental group was taught by a postdoctoral fellow using instruction based on research on learning. The same selected learning objectives were covered by both instructors in a 1-week period.

The instructional design for the experimental section was based on the concept of "deliberate practice" (11) for the development of expertise.

${ }^{1}$ Carl Wieman Science Education Initiative, University of British Columbia, Vancouver, BC, Canada. ${ }^{2}$ Department of Physics and Astronomy, University of British Columbia, Vancouver, BC, Canada. ${ }^{*}$ On leave from the University of British Columbia and the University of Colorado.

†To whom correspondence should be addressed. E-mail: gilbertwieman@gmail.com

$\ddagger$ This work does not necessarily represent the views of the Office of Science and Technology Policy or the United States government.
The deliberate practice concept encompasses the educational ideas of constructivism and formative assessment. In our case, the deliberate practice takes the form of a series of challenging questions and tasks that require the students to practice physicistlike reasoning and problem solving during class time while provided with frequent feedback.

The design goal was to have the students spend all their time in class engaged in deliberate practice at "thinking scientifically" in the form of making and testing predictions and arguments about the relevant topics, solving problems, and critiquing their own reasoning and that of others. All of the activities are designed to fit together to support this goal, including moving the simple transfer of factual knowledge outside of class as much as possible and creating tasks and feedback that motivate students to become fully engaged. As the students work through these tasks, they receive feedback from fellow students (12) and from the instructor. We incorporate multiple "best instructional practices," but we believe the educational benefit does not come primarily

from any particular practice but rather from the integration into the overall deliberate practice framework.

This study was carried out in the second term of the first-year physics sequence taken by all undergraduate engineering students at the University of British Columbia. This calculus-based course covers various standard topics in electricity and magnetism. The course enrollment was 850 students, who were divided among three sections. Each section had 3 hours of lecture per week. The lectures were held in a large theaterstyle lecture hall with fixed chairs behind benches grouping up to five students. The students also had weekly homework assignments, instructional laboratories, and tutorials and recitations where they solved problems; this work was graded. There were two midterm exams and a final exam. All course components were common across all three sections, except for the lectures, which were prepared and given independently by three different instructors.

During week 12, we studied two sections whose instructors agreed to participate. For the 11 weeks preceding the study, both sections were taught in a similar manner by two instructors (A and B), both with above average student teaching evaluations and many years experience teaching this course and many others. Both instructors lectured using PowerPoint slides to present content and example problems and also showed demonstrations. Meanwhile, the students took notes. "Clicker" (or "personal response system") questions (average 1.5 per class, range 0 to 5 ) were used for summative evaluation (which was characterized by individual testing without discussion or follow-up other than a summary of the correct answers). Students were given participation credit for submitting answers.

Before the experiment, a variety of data were collected on the students in the two sections

Table 1. Measures of student perceptions, behaviors, and knowledge.

Control section Experimental section

\begin{tabular}{lcc}
\hline Number of students enrolled & 267 & 271 \\
Mean BEMA score (13) (week 11) & $47 \pm 1 \%$ & $47 \pm 1 \%$ \\
$\begin{array}{l}\text { Mean CLASS score (14) (start of term) } \\
\quad \text { (agreement with physicist) }\end{array}$ & $63 \pm 1 \%$ & $65 \pm 1 \%$ \\
Mean midterm 1 score & $59 \pm 1 \%$ & $59 \pm 1 \%$ \\
Mean midterm 2 score & $51 \pm 1 \%$ & $53 \pm 1 \%$ \\
Attendance before experiment* & $55 \pm 3 \%$ & $57 \pm 2 \%$ \\
Attendance during experiment & $53 \pm 3 \%$ & $75 \pm 5 \%$ \\
Engagement before experiment* & $45 \pm 5 \%$ & $45 \pm 5 \%$ \\
Engagement during experiment & $45 \pm 5 \%$ & $85 \pm 5 \%$ \\
\hline
\end{tabular}

${ }^{\star}$ Average value of multiple measurements carried out in a 2-week interval before the experiment. Engagement also varies over location in the classroom; numbers given are spatial and temporal averages. 